\title{
Sample Management Tools for the Irradiation Test Area
}

\section{Morgan Logsdon, College of William and Mary}

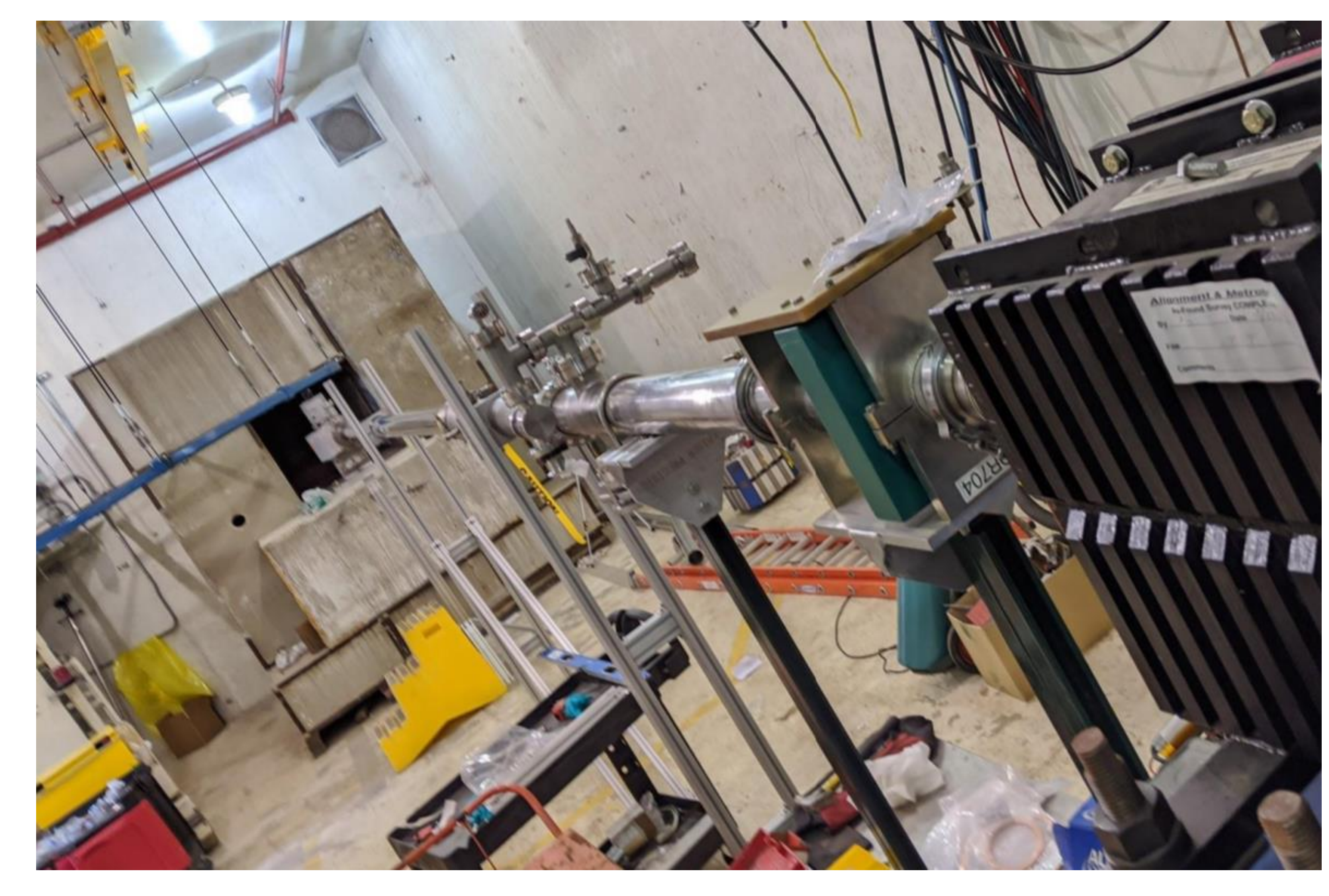

Photo from ITA Beam Line Installation

\section{Irradiation Test Area (ITA)}

The ITA offers onsite radiation testing, and will be available starting Fall 2020. It is projected to have testing slots about 40x/year and a capacity to reach fluences of $10^{16}$ protons $/ \mathrm{cm}^{2}$ in four hours. A $400 \mathrm{MeV}$ proton beam is directed to samples in a concrete shielding cave, with variations in place available to the user's preferences. The ITA regulates all its experiment data through ITA's Data Manager (IDM).

\section{ITA Data Manager (IDM) Functions}

For ITA users, the IDM provides organization and presentation of test results as well as coordination of logistics with administrators. It also allows for users to compare and view experiments and results. For administrators, the IDM allows regulation of user access, calculation of activation and cool-off times, and tracking for sample location and chain of custody.

\section{IDM at Fermilab}

Fermilab's IDM is an adaptation of CERN's IRRAD Data Manager. The original IDM was previously in use in the Proton Irradiation Facility (IRRAD) at CERN, though the facility is currently shut down.

The IDM is developed in a Python-based web-development framework called Django. It offers web-based data management and a built-in database securely stores beamspecific values used in activation/cool-off time calculations.

More information on original IDM: http://icalepcs2019.vrws.de/papers/mopha048.pdf

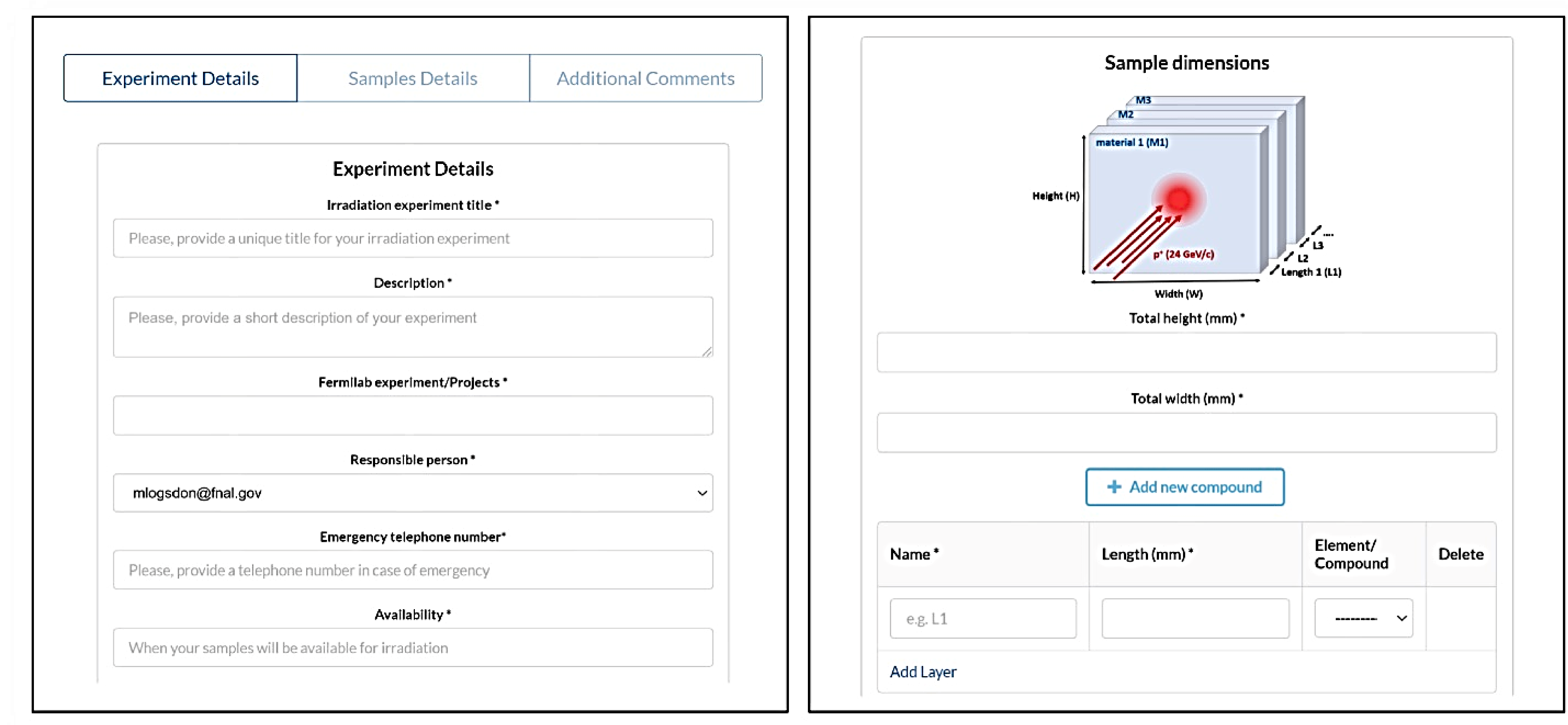

Screenshots from Django forms in IDM; New Experiment (left) and New Sample (right)

\section{IDM Stages of Development}

The IDM underwent three main phases of development before its deployment phase (ongoing). In the first phase, its functions and capabilities were documented and the backend code files were catalogued. In the second phase changes were made to IDM content based on ITA needs, and code was commented and edited in preparation for future development. In the third phase the database content was edited so that stopping power values reflected ITA needs.

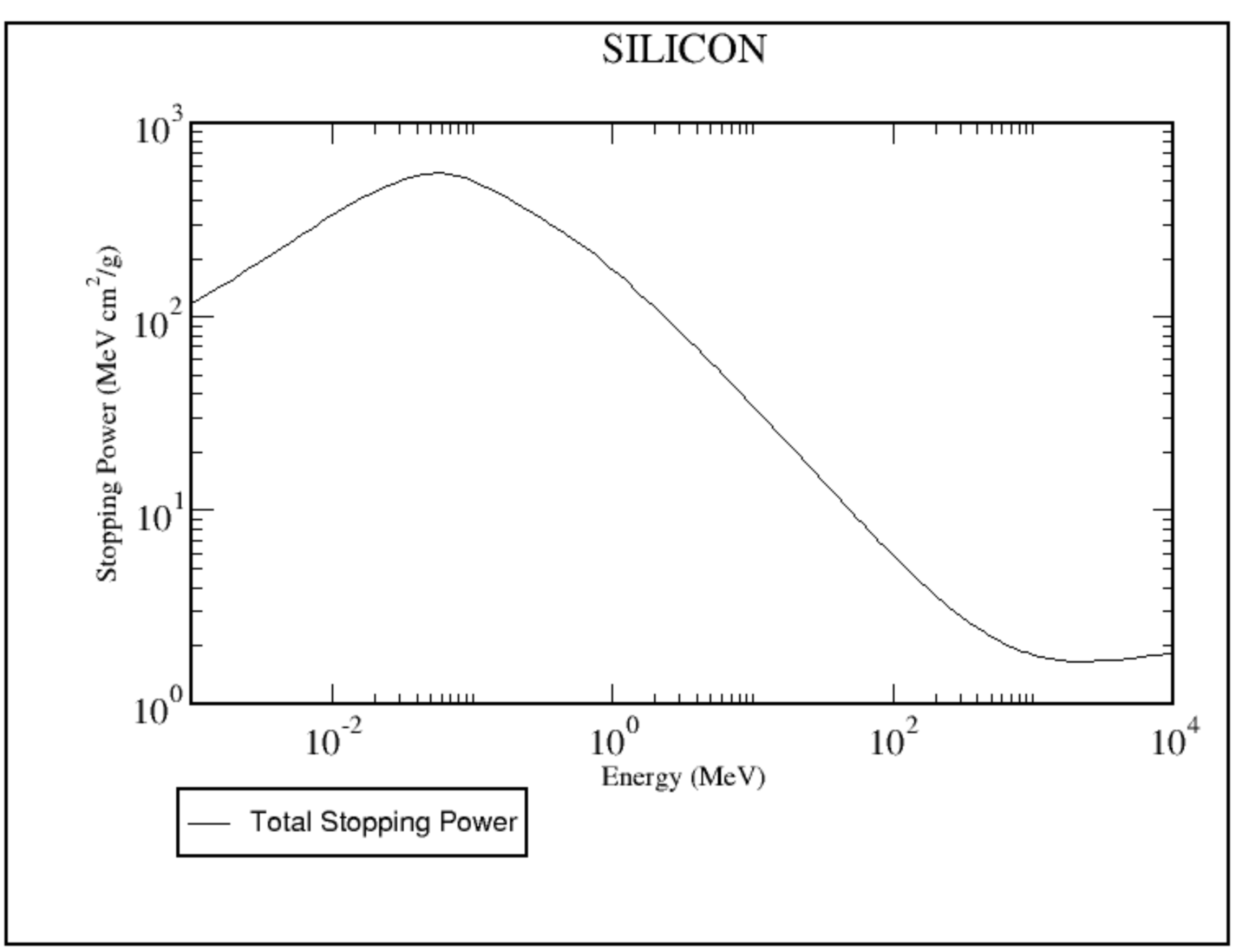

Stopping Power vs. Proton Energy from pstar (Stopping Power and Range
Tables for Protons) by the National Institute of Standards and Technology (NIST) https://physics.nist.gov/PhysRefData/Star/Text/PSTAR.html

\section{Stopping Power (dE/dx) Calculations}

Stopping power is the measure of energy transfer to a medium by a charged particle (ITA protons). For ITA purposes, it quantifies how much energy from the $400 \mathrm{MeV}$ protons that sample absorbs. The IDM database contained these calculations for CERN's beams, but now contains a full list of values for 97 elements calculated for the ITA proton beam. Early ITA use to test CMS, ATLAS chips (mostly silicon).

$$
-\frac{d E}{d x}=0.3071 z^{2} \frac{Z}{A} \frac{1}{\beta^{2}} \log \frac{\left(1.022 \times 10^{6}\right) \gamma^{2} \beta^{2}}{I}-\beta^{2}-\log \gamma
$$

Stopping power, measured in $\mathrm{MeV} \mathrm{cm}^{2} \mathrm{~g}^{-1} . Z$ and $A$ refer to properties of the number of the neam particle, the ratio of the perticle $z$, 6 , and $v$ are the atomic light, and a 6 -dependent constant. $/$ is the excitation energy, dependent on the atomic number of the medium. 